\title{
Mueller polarimetric imaging of biological tissues: classification in a decision-theoretic framework
}

\author{
Christian Heinrich ${ }^{1, *}$, Jean Rehbinder ${ }^{1}$, André Nazac $^{2}$, Benjamin Teig $^{3}$, \\ Angelo Pierangelo ${ }^{4}$, And Jihad Zallat ${ }^{1}$ \\ ${ }^{1}$ ICube, Université de Strasbourg, CNRS (UMR 7357), 300 bld. S. Brant, CS 10413, 67412 Illkirch cedex, France \\ ${ }^{2}$ Department of Obstetrics and Gynecology, University Hospital Brugmann, Université Libre de Bruxelles, Bruxelles, Belgium \\ ${ }^{3}$ Bicêtre Hospital, AP-HP, Department of Anatomopathology, Le Kremlin-Bicêtre, France \\ ${ }^{4}$ LPICM, CNRS, Ecole Polytechnique, Université Paris Saclay, Palaiseau, France \\ *Corresponding author: christian.heinrich@unistra.fr
}

Compiled October 13, 2018

\begin{abstract}
Mueller polarimetry is increasingly recognized as a powerful modality in biomedical imaging. Nevertheless, principled statistical analysis procedures are still lacking in this field. This paper presents a complete pipeline for polarimetric bioimages, with an application to ex vivo cervical precancer detection. In the preprocessing stage, we evaluate the replacement of pixels by superpixels. In the analysis stage, we resort to decision theory to select and tune a classifier. Performances of the retained classifier are evaluated. Decision theory provides a rigorous and versatile framework, allowing generalization to other pathologies, to other imaging procedures, and to classification problems involving more than two classes.
\end{abstract}

(c) 2018 Optical Society of America

http://dx.doi.org/10.1364/ao.XX.XXXXXX

\section{INTRODUCTION}

Optical tissue diagnosis is an important tool for the clinician, because of its low cost, non invasiveness, and high sensitivity allowing early detection. This technique allows to define reliable and pertinent resection margins in case of surgery.

In the realm of optical imaging techniques, Mueller polarimetry stands as a powerful modality delivering thorough information related to the biological phenomena at stake or the physical structure of materials. Generally speaking, polarimetric images provide information which is lacking in intensity images. Mueller polarimetric imaging has for example been successfully applied to cervical precancer diagnosis [1, 2], tissue diagnosis (see [3, 4], and see e.g., [5] for a broader point of view), skin probing [6], and materials classification [7-10].

Given the 16 parameters of the Mueller matrix (multiplied by the number of wavelengths at which the measurements are carried out), the full power of Mueller imaging is achieved only when a proper identification of the polarimetric signatures is performed (for example of healthy tissue versus diseased tissue). There is still a gap between Mueller imaging devices, which have been given much scientific interest, on the one hand, and data processing and statistical analysis of Mueller measurements on the other hand. Several conventional classification strategies have been applied to Mueller data, including linear classifiers (linear discriminant analysis and principal component analy- sis), classification trees, support vector machines, and partial least squares (see e.g., [1, 7, 10-12]). The article by Vaughn et al. indicates the need for a nonlinear classification scheme, and proposes nonlinear support vector machines over nonlinear Mueller parameters [10]. The article by Hoover et al. proposes a nonlinear modeling of principal components [7]. Strategies related to model training and classification can also be found in some works (see e.g., the case of support vector machines [10], with nested cross-validation for training, and empirical risk minimization for model selection).

In this work, we are proposing a global statistical framework based on decision theory, which may be generalized and used in any biological tissue diagnostic case or materials classification problem. Nonlinear classification, nested cross-validation, empirical risk minimization, and weighting the probability of errors relatively to the importance of those errors are features of the proposed approach. Our main contributions for the preprocessing aspect are the incorporation of physical admissibility constraints and the use of superpixels to tackle information redundancy in the vast amount of data available. On the classification side, we propose a statistically loss-risk based approach combined with various machine learning tools, to differentiate normal tissue from precancerous one. Grounding the algorithm on such a decision-theoretic basis allows to address cases where more than two labels are at stake. We provide tools to identify 
polarimetric biomarkers and to select automatically the optimal classifier among a given set. Performance scores that might be expected on unseen tissues are computed. The pathology of interest in this article is cervical cancer, but the preprocessing and classification procedure proposed may be applied to a much broader class of pathologies.

In this article, we propose a low-level approach where the entity of interest is the pixel or the superpixel. This is to be opposed to higher level approaches, where the goal is to identify whether cancer or precancer is present in an entire image, or in an image tile, or in an image patch (see e.g., $[13,14]$ ). The methodology proposed here might be considered for future incorporation in higher level approaches.

This article is structured as follows: the next section describes the imaging setup and the image database. The third section deals with the data preprocessing part of the pipeline. The fourth section addresses the classification procedure adopted. Results are detailed in the fifth section. Conclusions are drawn and perspectives given in the final section.

\section{IMAGING SETUP AND DATABASE}

The multispectral imaging Mueller polarimeter in backscattering configuration used for the ex vivo analysis of specimens of the uterine cervix is detailed in [1]. It has been designed according to the results of a case study published in 2013 [15]. This case study demonstrated that polarimetric imaging in the visible range holds potential for distinction between healthy and precancerous tissue. Shorter wavelengths probe areas closer to the epithelium (where the infection by HPV responsible for cervical cancer takes place) whereas longer wavelengths give insights in the underlying tissue architecture. Taking advantage of this case study, three different wavelengths $(450,550$, and $600 \mathrm{~nm}$ ) have been chosen for the present investigation.

The current study includes a total of twenty three conization specimens obtained as part of a larger study conducted in collaboration with the University Hospital of Kremlin-Bicêtre. Patients included in the study have been scheduled for a preventive surgical excision of the uterine cervix following a standard medical procedure (e.g., after detection of abnormal cells by the Pap smear followed by detection of a high-grade lesion by colposcopy and biopsy). Measurements on the surgical samples were performed in the department of anatomic pathology after fixation in formalin and prior to further processing by clinicians according to the conventional protocol. Fixation time was set to 24 hours after reception of the fresh sample in the department.

As described in [1], a special procedure has been designed yielding an accurate histopathological mapping of the precancerous lesions in the polarimetric images. This mapping will constitute the ground truth for the present study. On the 23 tissues available, 56 zones of interest have been delineated and labeled. A given zone comprises from 900 to 7000 pixels, corresponding to 20 to 250 groups of similar pixels, named superpixels (see Fig. 3). All pixels of a given zone have been tagged with the same label by the clinician.

As in [1], the aim of this study is to differentiate high-grade cervical intraepithelial neoplasia (CIN 2-3) from healthy squamous epithelium (EMS).

\section{DATA PREPROCESSING}

In the Mueller imaging framework, the noisy raw radiance $\boldsymbol{I}(s)$ acquired at pixel $s$ is linked to the $16 \times 16$ polarization Mueller matrix $($ PMM) $\boldsymbol{P}$ and the unknown $16 \times 1$ vectorized Mueller matrix $\underline{M}^{\star}(s)$ at pixel s, obtained by stacking the columns of the $4 \times 4$ Mueller matrix $\boldsymbol{M}^{\star}(s)$, by the observation equation

$$
\boldsymbol{I}(s)=\boldsymbol{P} \underline{\boldsymbol{M}}^{\star}(s)+\boldsymbol{\varepsilon}^{\star}(s),
$$

where $\varepsilon^{\star}(s)$ accounts for (unknown) observation noise and modeling errors. Radiance is acquired at three different wavelengths [1]. The wavelength is not mentioned in the observation equation for the sake of clarity.

Mueller parameter estimation is carried out using pseudoinverse under admissibility constraints, as

$$
\underline{\widehat{\boldsymbol{M}}}(s)=\arg \min _{\underline{\boldsymbol{M}} \in \mathcal{M}}\|\boldsymbol{I}(s)-\boldsymbol{P} \underline{\boldsymbol{M}}\|^{2},
$$

where $\mathcal{M}$ denotes the set of physically admissible vectorized Mueller matrices. The reader is referred to [16] for details about the optimization procedure used. Estimation is carried out independently for each wavelength.

At this step, pixels exhibiting a depolarization power lower than 0.6 at $550 \mathrm{~nm}$ are set aside from the study, since they are considered as being contaminated by specular reflection (see also [1]).

Since the amount of pixels at stake is enormous whereas the field of Mueller matrices exhibits redundancy, data reduction can be taken advantage of. We chose to group similar neighboring pixels in superpixels [17, 18]. Generally speaking, superpixels adhere to boundaries. In the present work, superpixels are determined considering all Mueller channels and all wavelengths jointly. Thereby, only pixels with a similar behavior across all wavelengths are grouped. Besides, such a grouping is achieved zone by zone, thus ensuring that all grouped pixels share the same label. This comes at negligible information loss, and does not smooth boundaries. In practice, this allowed us to reduce the volume of data by a factor 27.

Each superpixel is now equipped with three physically admissible Mueller matrices (one matrix for each wavelength). To enhance the performances of the forthcoming classification procedure and give the classifier more grip onto the data, we supplemented each superpixel with physical parameters stemming from the Mueller matrix: four parameters (scalar retardance, linear retardance, depolarization, and orientation of the birefringence slow axis) were incorporated for each wavelength, thus yielding $3 \times(16-1+4)=57$ parameters for each superpixel (the latter " 1 " results from the exclusion of the first coefficient of Mueller matrices, which is always equal to one as a result of normalization). Those nonlinear additional physical parameters may also improve the physical interpretability of the retained classifiers and shed light on the physical phenomena at stake. The resulting vector of so-called Mueller parameters is denoted $x_{n}$ in the sequel, where $n$ indexes the superpixel.

A flowchart of the global processing pipeline is given in Fig. 1.

\section{CLASSIFICATION PROCEDURE}

The problem at hand now is a classification one. Considering a measurement set $\left\{\left(x_{n}, c_{n}\right), n=1, \ldots N\right\}$ of Mueller parameters $x_{n}$ and labels $c_{n}$, where $N$ is the total number of superpixels, the goal is to select automatically a classifier (a decision function) $\widehat{c}: x \longmapsto \widehat{c}(x)$ able to classify an unseen vector $x$ of parameters.

A confusion matrix point of view analysis sheds interesting light on the proposed classification procedure. The goal is to link the approach with specificity and sensitivity scores, as well as with classification accuracy and average class accuracy, which 


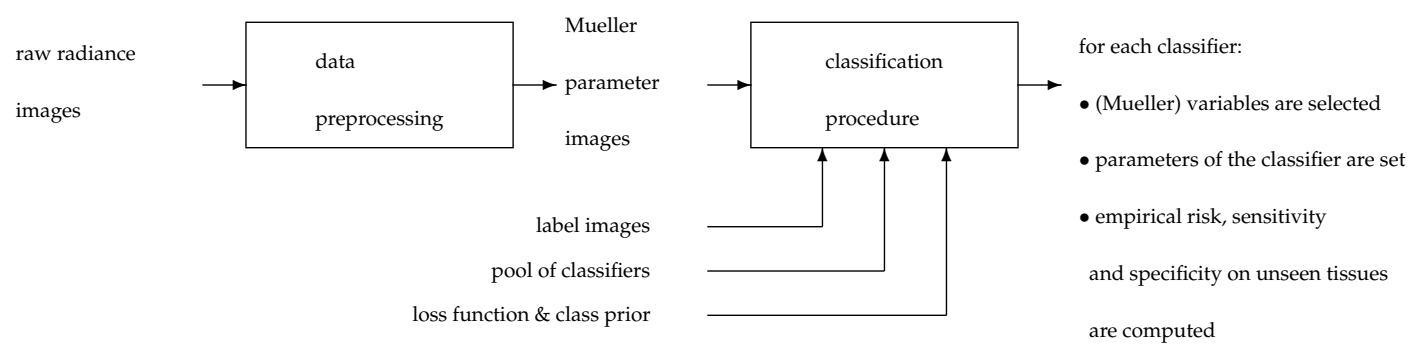

Fig. 1. Flowchart of the general processing pipeline. The classification procedure is detailed in Fig. 2.

are classically used in classifier assessment. Such an analysis is proposed in the appendix section, at the end of the article.

\section{A. Loss and risk}

In a decision-theoretic approach, a loss function $\ell(a, b)$ has to be considered, where $\ell$ is the cost incurred if $a$ is the true label (the true class), and $b$ is the predicted label, issued by the classifier. A loss $\ell$ may be considered as a realization of the random variable $L$. The rationale for choosing the most pertinent classifier is to minimize the risk $\mathcal{R}=\mathbb{E}[L]$ (see e.g., $[19,20]$ ). Risk minimization allows to select a classifier among a set of candidates. It also allows to determine the optimal set of variables (i.e., of Mueller parameters) and to learn the parameter values for a given classifier.

The risk writes

$$
\mathcal{R}=\mathbb{E}[L]=\sum_{c} \int_{x} p(X=x, C=c) \ell(c, \widehat{c}(x)) \mathrm{d} x,
$$

where $c$ is the true label and $\widehat{c}(x)$ the label issued from the classifier. In practice, the distribution $p(X=x, C=c)$ is unknown and has to be learnt from available data.

We consider that each labeled tissue sample bears partial information about the underlying distribution. This information comes in the form of samples $\left(\boldsymbol{x}_{n}, c_{n}\right)$. The realization $c_{n}$ is constant on a zone. A tissue sample may encompass different zones, each zone having its own label. Different labels may therefore be present in a given tissue sample. Moreover, we suppose $p(C=c)$ to be uniformly distributed among labels: we do not assume that the prior distribution $p(C=c)$ is well represented by the proportions of labels contained in the measurement set. The reasons motivating this choice are detailed in the sequel. If needed, this prior distribution can be set to fit any available knowledge.

We want to avoid that tissues encompassing a lot of pixels as a result of labeling by the expert excessively dominate tissues where much less pixels have been labeled. Such a situation may lower the detection ability of the classifier, and may finally impair the global performance. Restoring balance can be addressed by resampling (see e.g., [10]). Here, we propose a reweighting approach, by giving equal weight to all tissue samples: when estimating $p(\boldsymbol{X}=\boldsymbol{x} \mid \mathrm{C}=c)$ from available data, a tissue sample having many superpixels of a given label will not contribute more than another tissue sample having few superpixels of the same label. The weight (the contribution) of a given superpixel of a given tissue sample will therefore be inversely proportional to the number of superpixels of the same label in this tissue. The rationale here is that a small zone with a typical and rare signature should not be overwhelmed during the learning phase by large zones with signatures occuring more frequently. Such an enhancement of rare signatures will improve future identification of similar signatures presented to the classifier. If overwhelmed by different signatures of the same class, rare signatures may be left in a zone of the parameter space far from the bulk of signatures. In such a case, the classification will be less reliable, as being an issue of extrapolation from classical and typical signatures related to different tissue types, with an increased possibility of misclassification.

\section{B. Empirical risk}

The risk $\mathcal{R}$ also writes

$$
\mathcal{R}=\sum_{c} p(C=c)\left[\int_{x} p(X=x \mid C=c) \ell(c, \widehat{c}(x)) \mathrm{d} x\right],
$$

where the part $\int_{x} p(X=x \mid C=c) \ell(c, \widehat{c}(x)) \mathrm{d} x$ - the risk conditioned on $C-$ will have to be evaluated using available data. For tissue sample $i_{s}$, we define the conditional empirical risk $r_{e}\left(i_{s} \mid c\right)$ as

$$
r_{e}\left(i_{s} \mid c\right)=\frac{1}{\left|\mathcal{P}\left(i_{s}, c\right)\right|} \sum_{x \in \mathcal{P}\left(i_{s}, c\right)} \ell(c, \widehat{c}(x)),
$$

where $\mathcal{P}\left(i_{s}, c\right)$ denotes the set of superpixels in tissue sample $i_{S}$ with true label $c$, and $|\mathcal{P}|$ denotes the cardinal of the set $\mathcal{P}$. If label $c^{\prime}$ is not represented in a tissue sample, its associated risk $r_{e}\left(i_{s} \mid c^{\prime}\right)$ will be left undefined.

Within the framework defined above, the risk $\mathcal{R}$ will be approximated by the empirical risk $r_{e}$ :

$$
\begin{aligned}
\mathcal{R} & =\sum_{c} p(C=c)\left[\int_{x} p(\boldsymbol{X}=x \mid C=c) \ell(c, \widehat{c}(\boldsymbol{x})) \mathrm{d} x\right] \\
& \simeq r_{e}=\sum_{c} p(C=c)\left\langle r_{e}\left(i_{s} \mid c\right)\right\rangle_{i_{s} \in \mathcal{S}}
\end{aligned}
$$

where $\mathcal{S}$ is a set of tissue samples and where $\left\langle z_{k}\right\rangle_{k \in \mathcal{K}}$ is the empirical average of the coefficients $\left\{z_{k}, k \in \mathcal{K}\right\}$ (undefined values are left out in the computation of the empirical average). The rationale is that each tissue sample contributes equally to approximating $\int_{x} p(X=x \mid C=c) \ell(c, \widehat{c}(x)) \mathrm{d} x$ for class $c$, when this class is represented. The contribution of one given tissue sample is $r_{e}\left(i_{s} \mid c\right)$. Since all tissue samples contribute with the same weight, the overall contribution writes $\left\langle r_{e}\left(i_{S} \mid c\right)\right\rangle_{i_{s} \in \mathcal{S}}$.

In the present study, we considered $\ell(a, b)=1-\delta_{a, b}$, where $\delta_{a, b}$ is the Kronecker delta. A false positive thus incurs the same loss as a false negative. In practice, especially in the case of biological and medical diagnosis, a false negative may be worse than a false positive. This can easily be accounted for in the loss $\ell(a, b)$. With $\ell(a, b)=1-\delta_{a, b}, r_{e}\left(i_{s} \mid c\right)$ is the empirical misclassification rate for true label $c$ in tissue sample $i_{s}$. 
We would like to emphasize that the empirical risk $r_{e}$ does not correspond to the average misclassification rate computed across all superpixels regardless of classes. There are two reasons for that. Firstly, when computing $\left\langle r_{e}\left(i_{s} \mid c\right)\right\rangle_{i_{s} \in \mathcal{S}}$ and thus when computing $r_{e}$, each tissue sample contributes with the same weight, whereas in the case of average misclassification rate across superpixels, the relative influence of a tissue sample depends on the number of its superpixels. As an extreme example, one may think of a tissue sample having one single superpixel, supposed to be misclassified. This tissue sample will play almost no role and have no influence, when facing another sample having tens of superpixels. In the proposed framework, the first tissue will have the same influence as if all superpixels of the second tissue were misclassified. Secondly, when computing $r_{e}, p(C=c)$ is imposed by the model, whereas in the case of average misclassification rate across superpixels it is imposed by the data and might therefore not correspond to its theoretical or wished value.

Enforcing and expliciting such normalizing procedures (normalization $\left\langle r_{e}\left(i_{s} \mid c\right)\right\rangle_{i_{s} \in \mathcal{S}}$ across samples conditioned on classes and normalization across classes by imposing $p(C=c))$ is also of interest to the clinician and to the data scientist. This allows for example to apprehend clearly what effect it has to delineate a large zone in a given sample, and a small zone in another sample: the clinician should not refrain from delineating a large zone in a given tissue, since this will not wash out a smaller zone in another sample.

\section{Variable selection, parameter setting, classifier assess- ment}

This section addresses variable selection, parameter setting, and classifier assessment aspects of the procedure. The outline is given in the first subsection. Details are dealt with in the following subsections.

\section{C.1. Procedure outline and retained cross-validation scheme}

The final classifier will use only a subset of the entire set of Mueller parameters available, since including irrelevant or redundant parameters in a classifier degrades its overall performance. Variable selection will thus have to be incorporated in the procedure. Moreover, for some classifiers, parameters may have to be set, as for example the number of nearest neighbors for a nearest neighbor classifier, or the border for a linear discriminant. Finally, we want to assess model performance (classifier performance), in order to give the investigator an idea of the performance that may be expected on new (incoming) unlabeled data.

All this will be achieved using nested cross-validation (CV). Inner CV will allow variable selection and parameter setting. Outer CV will allow model performance assessment.

For the $i_{t}^{\text {th }}$ test run out of a total of $I_{t}$ test runs, the set of tissue samples will be split (outer $\mathrm{CV}$ ) between test samples, and train \& validate samples. A $50: 25: 25$ proportion split for train-validate-test is recommended in [21]. We worked with a $12: 6: 5$ split on the 23 available tissue samples. The $12: 6$ split corresponds to 3 -fold CV (inner CV), the set of 18 train \& validate samples being split in 3 parts. Each part is successively used as a validation set, whereas samples from the parts left are used for training. The 3-fold approach is imposed by the $50: 25: 25$ proportion split.

We used stratified sampling: in each subset (train, validate, and test), the proportion of labels is roughly equal to the proportion of labels present in the global set.
An example of tissue samples splits is given in Table 1.

We used a wrapper approach to select the useful Mueller parameters: the machine learning algorithm itself will select the variables, the learning algorithm being wrapped in the selection procedure. A subset of features is thus evaluated based on the accuracy of prediction using that subset. This is to be opposed to filtering approaches, where the subset of variables is determined before learning begins. We used forward selection: the variables are incorporated one by one, in an incremental greedy search procedure. The procedure is detailed in the sequel.

Once the variables are selected and the parameters of the classifier set, the model is retrained on the train \& validate set of tissue samples. The model is then used to predict labels on the test set of tissue samples. Thus, test samples are unseen in the whole learning process, which is important to give the investigator a fair point of view on the quality of prediction to be expected on new data.

An overview of the classification procedure is given in Fig. 2.

For any type of classifier a wide range of complexity degrees is evaluated on the training set of tissues (e.g., the number of neighbors for the nearest neighbor classifier spans a large set of values, the complexity of classifier trees examined varies from very simple to very complex). Assessment on the validation set determines the appropriate degree of fitting, thus precluding underfitting and overfitting.

Ideally, if data were plentiful, training, testing, and validating would all be done on separate sets of tissues. This would be equivalent to having one single test run, and to have tissues dedicated to training, whereas other tissues would be dedicated to validating. But we are in a situation of scarce data. Validating on a set of completely untouched tissues would yield a assessment score with high variability, one realization of which would not be very informative.

\section{C.2. Model fitting}

Model fitting (classifier fitting, classifier training) takes place on the training set of tissue samples (with a view to variable selection and parameter setting, inner $\mathrm{CV}$ ) and on the training and validation set of tissue samples (with a view to classifier assessment, outer $\mathrm{CV}$ ).

Each sample $\left(x_{n}, c_{n}\right)$ used for fitting the model is given a weight (see sections $4 \mathrm{~A}$ and $4 \mathrm{~B}$ ). This weight is incorporated in the model fitting phase. The class prior $p(C=c)$ and the loss $\ell$ are used during the model fitting phase as well.

The output of model fitting is a set of classifier parameters values, such as for example the architecture (nodes and thresholds) of a classification tree, or the border for a linear classifier.

\section{C.3. Model assessment}

Model assessment (classifier assessment) takes place on the validation set of tissue samples using the model fit on the training set of tissue samples (with a view to variable selection and parameter setting, inner $\mathrm{CV}$ ) or on the testing set of tissue samples using the model fit on the training and validation set of tissue samples (with a view to classifier assessment, outer $\mathrm{CV}$ ).

Let us consider first the case of model assessment on the validation set (inner CV model assessment). We work here with 3-fold CV (see above). The model is fit on the training set, prediction is made on the validation set. Prediction is made successively on each fold, thus generating at the end one prediction for each tissue present in the train \& validation set. Model assessment is based on this set of predictions. The predictions 
Table 1. Example of the Beginning of a List of Train-Validate-Test Sample Sets of Tissue Samples. Format: [Train-Validate Fold 1] [Train-Validate Fold 2] [Train-Validate Fold 3] - [Test].

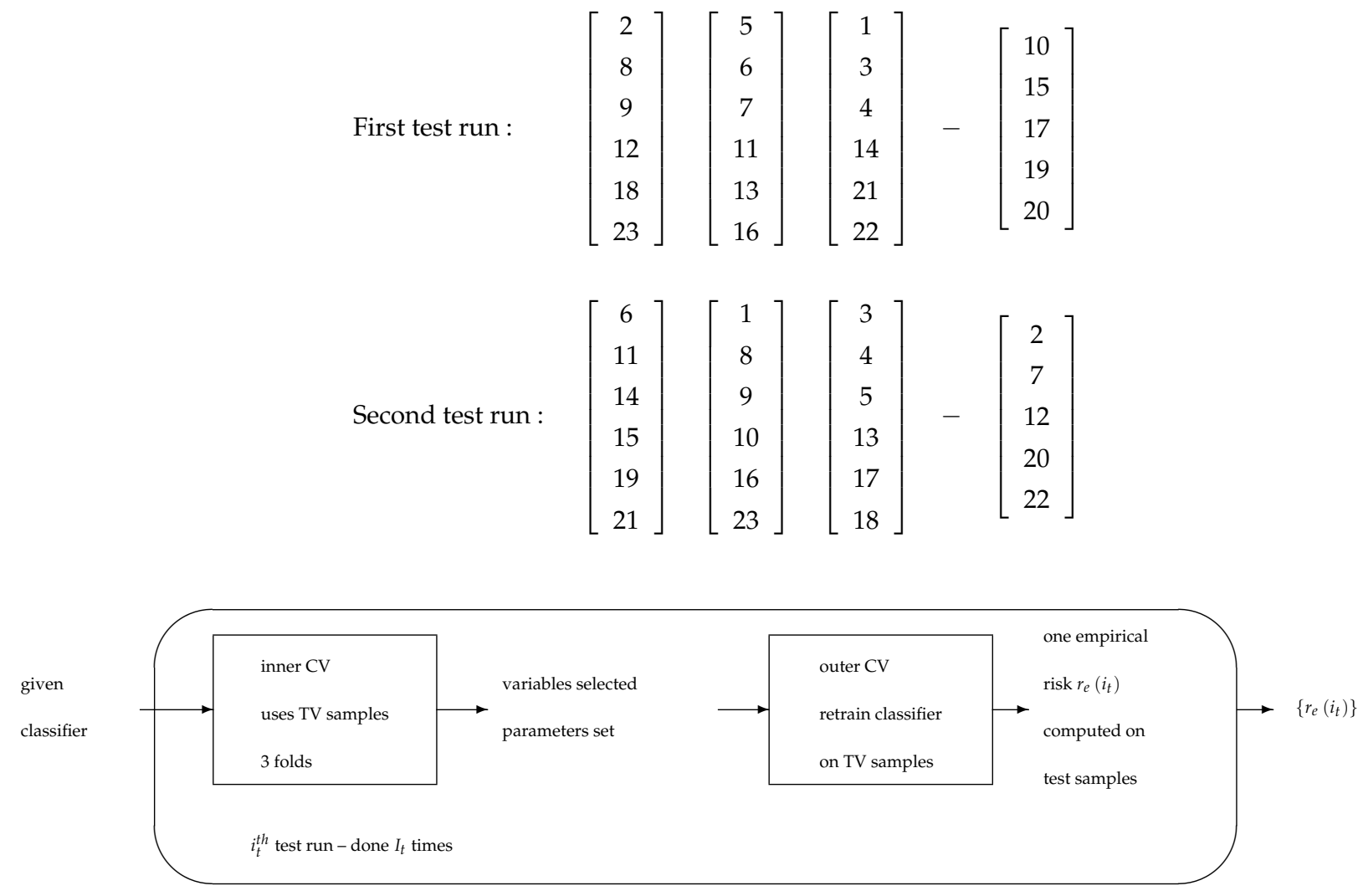

Fig. 2. Flowchart of the classification procedure, as a part of the global pipeline (see Fig. 1). Mueller parameter images, label images, loss function and class priors are also inputs of the pipeline, besides the given classifier. TV samples stands for train and validate tissue samples.

being supposed to be known, we focus now on the assessment part.

Let $\mathcal{S}_{\mathrm{TV}\left(i_{t}\right)}$ be the set of tissue samples used for training and validation in the $i_{t}^{\text {th }}$ test run. For any tissue $i_{S} \in \mathcal{S}_{\mathrm{TV}\left(i_{t}\right)}$, we predict labels $\widehat{c}\left(i_{s}, n\right)$, where $n$ is indexing the superpixels in $i_{s}$. For sample $i_{s}$, and using the predictions $\widehat{c}\left(i_{s}, n\right)$, we compute the empirical risk $r_{e}\left(i_{s} \mid c\right)$ related to class $c$ (more than one class may be present in $i_{s}$, yielding several empirical risks) as

$$
r_{e}\left(i_{s} \mid c\right)=\frac{1}{\left|\mathcal{S}\left(i_{s}, c\right)\right|} \sum_{n \in \mathcal{S}\left(i_{s}, c\right)} \ell\left(c, \widehat{c}\left(i_{s}, n\right)\right) .
$$

The resulting score (i.e., the empirical risk) for assessment (inner $\mathrm{CV}$ assessment) for the $i_{t}^{\text {th }}$ test run writes

$$
r_{e}=\sum_{c} p(C=c)\left\langle r_{e}\left(i_{s} \mid c\right)\right\rangle_{i_{s} \in \mathcal{S}_{\mathrm{TV}\left(i_{t}\right)}} .
$$

We now address model assessment on the testing set. Model assessment on the testing set is similar to model assessment on the validation set. The model is retrained on the whole train \& validate set of tissues, prediction is made on the testing set of tissue samples. The testing set of tissue samples depends on the index $i_{t}$ of the test run.
The empirical risk at stake here writes

$$
r_{e}\left(i_{t}\right)=\sum_{c} p(C=c)\left\langle r_{e}\left(i_{s} \mid c\right)\right\rangle_{i_{s} \in \mathcal{S}_{\mathrm{TST}\left(i_{t}\right)}},
$$

where $\mathcal{S}_{\mathrm{TST}\left(i_{t}\right)}$ is the testing set of tissue samples for the $i_{t}^{\text {th }}$ test run. We thus get a set of $I_{t}$ empirical risks, one risk for each test run. This set may be represented by different statistics (mean, median, standard deviation, inter-quartile range, etc). Those statistics will be used to compare different classifiers, such as for example $k$-nearest neighbors (KNN) versus linear discriminant.

\section{C.4. Illustration of variable selection and parameter setting}

As an illustration of the variable selection (predictor determination) and parameter setting procedure, let us take the example of KNN classification. We consider here, for the sake of clarity, the simple case of a set of two different models: $\mathcal{M}_{1}$ is the first model, using one neighbor for classification, $\mathcal{M}_{2}$ is the second model, using three neighbors for classification. On a given train $\&$ validate tissue sample set, the goal is to select the pertinent set of variables (Mueller parameters) and to choose between $\mathcal{M}_{1}$ and $\mathcal{M}_{2}$.

For model $\mathcal{M}_{1}$, let us suppose that $n_{v}$ variables have been incorporated so far. We try to include one more variable. To this end, we consider incorporating any left out variable (we consider each left out variable, separately, one by one). We 
compute the empirical risk $r_{e}$ corresponding to incorporating one particular left out variable (see Eq. (8)). The minimum value of this set of risks (one risk for each left out variable) is considered. If this minimum risk outperforms the risk $r_{e}\left(n_{v}\right)$ with $n_{v}$ variables by at least a threshold $\tau$ (i.e., is lower than $\left.r_{e}\left(n_{v}\right)-\tau\right)$, the variable corresponding to the minimal risk is incorporated. Using a non null positive threshold may improve performance by avoiding overfitting. The process stops when no candidate variable can successfully be incorporated. A total of $N_{v}^{1}$ variables is supposed to be incorporated at the end of the process. The final risk $r_{e}\left(\mathcal{M}_{1}, N_{v}^{1}\right)$ is to be compared to $r_{e}\left(\mathcal{M}_{2}, N_{v}^{2}\right)$ for choosing the model.

\section{EXPERIMENTAL RESULTS}

We worked with a set of 23 tissue samples, encompassing 150000 pixels or 5550 superpixels (see Fig. 3), depending on the point of view. Polarimetric measures were taken at 450, 550, and $650 \mathrm{~nm}$. A total of $I_{t}=25$ test runs were considered for each experiment. We worked with the $12: 6: 5$ split mentioned above for the train:validation:test partition of samples.

In the first subsection, we deal with classification using scalar retardance at $450 \mathrm{~nm}$, as advised in [1]. We mainly address here the preprocessing part of the global pipeline. We evaluate different possible choices.

In the second subsection, we consider a wider class of polarimetric parameters, and a broader set of classifiers. The focus in this part is on the statistical analysis part of the pipeline. We consider a set of standard classifiers, whose performances are evaluated in conjunction with a variable selection procedure. Classifier selection is made between decision stump, classification tree, random forest, linear discriminant, naïve Bayes, $k$ nearest neighbors, support vector machine (SVM), and partial least squares (PLS, see e.g., [22, 23]).

We would like to underline the fact that in the entire analysis that will take place, the labeling (the ground-truth) is supposed to be perfect. This might not be the case, and as a consequence, the performances obtained here might underestimate the performances that could be obtained on perfectly labeled data.

\section{A. Classification using scalar retardance at $450 \mathrm{~nm}$ with a fo- cus on preprocessing}

Experiment 1: we pooled all pixels and determined the threshold yielding equal values for specificity and sensitivity. We got sensitivity $=$ specificity $=79.4 \%$, for a threshold of 8.90 . This is consistent with the results of [1], the slight variation in threshold, sensitivity, and specificity resulting from a variation in the data used (tissues have been added to the database since the work [1]). Besides, estimation is done here under physical admissibility constraints, which was not the case in [1]. This is also a source of variation.

Experiment 2: in the same framework as above, we consider superpixels instead of pixels. We get sensitivity $=$ specificity $=$ $81.3 \%$, for a threshold of 8.57 .

The results are improved using superpixels, along with a 27 times data volume reduction (there are 27 times less superpixels than pixels).

The problem in those experiments is that training and testing are achieved on the same dataset, which is known to yield overoptimistic results.
Experiment 3: to address the problem mentioned, we resort to cross-validation. On each test run, training is done on the train \& validate tissue sample set, assessment is done on the test tissue sample set. For each run, the threshold is set so as to enforce equality between specificity and sensitivity. All learning pixels are pooled and given the same weight. Prediction is now done about 865000 times (prediction can occur several times for a given pixel).

The thresholds belong to $[7.14 ; 10.29]$. If we pool all predictions in a single confusion matrix, we get: specificity $=76.7 \%$, sensitivity $=75.8 \%$.

The results can also be analyzed tissue sample by tissue sample. Since prediction can be made several times for a given tissue sample, we first average specificity (if defined) and sensitivity (if defined) across runs but for fixed tissue sample. In practice, such averages involve similar values, since the prediction quality for a given tissue sample does not vary much across runs. We then compute statistics on the set of specificities on one hand, on the set of sensitivities on the other hand. We denote respectively minimum, maximum, average, and quartiles as $m, M, a, q_{1}, q_{2}$, $q_{3}$. We get (in \%)

\begin{tabular}{|c|c|c|c|c|c|c|}
\hline & $m$ & $q_{1}$ & $q_{2}$ & $q_{3}$ & $M$ & $a$ \\
\hline specificity & 48.2 & 89.3 & 93.9 & 98.2 & 100 & 88.2 \\
sensitivity & 0.12 & 78.7 & 92.3 & 97.7 & 99.7 & 79.6 \\
\hline
\end{tabular}

As expected, we get results of lower quality than the results of experiment 1 (see the specificity and sensitivity extracted from the confusion matrix devised using pooled predictions, respectively $76.7 \%$ and $75.8 \%$, compared to $79.4 \%$ in experiment 1). Besides, using several statistics unveils interesting information, which remains hidden if using only average specificity and average sensitivity (column $a$ in the table). It appears that the median values $\left(q_{2}\right)$ are quite satisfying, and that some tissue samples are poorly labeled by the classifier (see $m$ and $q_{1}$, which are rather low). The presence of such tissues deteriorates the average values.

Experiment 4: the previous experiment is run on superpixels. Prediction is now done about 32000 times (prediction can occur several times for a given superpixel). The thresholds belong to [6.55; 10.08].

If we pool all predictions in a single confusion matrix, we get: specificity $=77.9 \%$, sensitivity $=76.2 \%$.

Analyzing the experiment tissue sample by tissue sample yields the following table

\begin{tabular}{|l|c|c|c|c|c|c|}
\hline & $m$ & $q_{1}$ & $q_{2}$ & $q_{3}$ & $M$ & $a$ \\
\hline specificity & 47.1 & 91.8 & 94.7 & 99.3 & 100 & 89.4 \\
sensitivity & 0 & 82.3 & 97.4 & 99.9 & 100 & 81.6 \\
\hline
\end{tabular}

Again we see that the use of superpixels improves the quality of the results. As before, some samples have very low quality predictions (see the $m$ values, and the $q_{1}$ value for sensitivity which are low as compared to $q_{2}$ scores).

Experiment 5: we now weight each superpixel in the learning phase. Weighting is done as detailed in section 4, Classification procedure: each superpixel is equipped with a weight inversely proportional to the number of superpixels in the same tissue sample with the same (true) label. Any tissue sample now has an influence similar to the one of any other tissue sample of the 


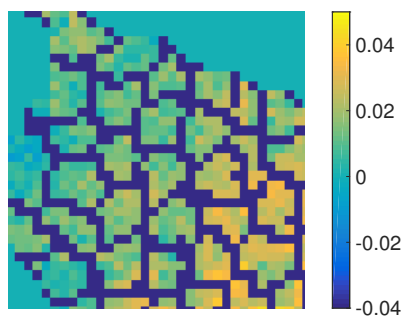

(a)

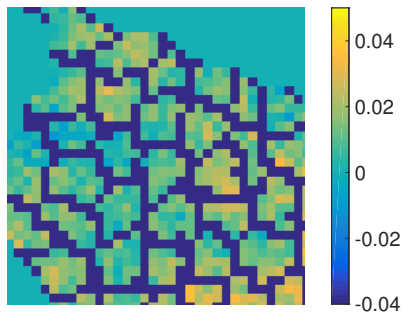

(b)

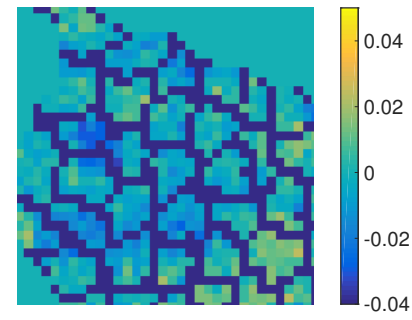

(c)

Fig. 3. Superpixels of a part of a zone of interest of one element of a Mueller matrix are represented here. View size: $79 \times 73$ pixels, $0.37 \times 0.34 \mathrm{~cm}$. (a) $450 \mathrm{~nm}$, (b) $550 \mathrm{~nm}$, (c) $600 \mathrm{~nm}$.

same (true) tissue label. The threshold is set such that specificity and sensitivity are equal on the training set.

If we pool all predictions in a single confusion matrix, we get: specificity $=74.3 \%$, sensitivity $=83.4 \%$. Analyzing the experiment tissue sample by tissue sample yields the following table

\begin{tabular}{|l|c|c|c|c|c|c|}
\hline & $m$ & $q_{1}$ & $q_{2}$ & $q_{3}$ & $M$ & $a$ \\
\hline specificity & 44.8 & 87.1 & 92.2 & 98.4 & 100 & 85.6 \\
sensitivity & 0 & 91.3 & 99.0 & 100 & 100 & 85.7 \\
$1-r_{e}$ & 62.0 & 86.5 & 89.2 & 93.0 & 97.2 & 86.6 \\
\hline
\end{tabular}

In this experiment and in the following ones as well, the statistics on $1-r_{e}$ are computed using the set $\left\{r e\left(i_{t}\right), i_{t}=1, \ldots I_{t}\right\}$ (see Eq. (9)).

Experiment 6: we start from experiment 5. Modification takes place on the threshold determination, which is set to minimize $r_{e}$, or equivalently to maximize $1-r_{e}$. This procedure is akin to the method proposed in this article. We chose $\ell(a, b)=1-\delta_{a, b}$. We get the following table

\begin{tabular}{|l|c|c|c|c|c|c|}
\hline & $m$ & $q_{1}$ & $q_{2}$ & $q_{3}$ & $M$ & $a$ \\
\hline specificity & 54.2 & 91.0 & 94.5 & 99.3 & 100 & 89.1 \\
sensitivity & 0 & 83.2 & 95.6 & 99.8 & 100 & 82.7 \\
$1-r_{e}$ & 61.6 & 86.5 & 89.7 & 94.1 & 97.7 & 86.9 \\
\hline
\end{tabular}

The figures in the $q_{2}$ columns in the two preceding tables may appear surprising at first sight, since the median $\left(q_{2}\right)$ value of $1-r_{e}$ is lower than the median value of both specificity and sensitivity, whereas $1-r_{e}$ may appear as an average of specificity and sensitivity (see the annex). In fact, the figures in the tables are right and can be explained.

On the one hand, each specificity and each sensitivity is computed tissue-wise. We thus get a set of values of specificity and a set of values of sensitivity. A lot of those values are high, since the corresponding samples are well labeled by the classifier. But some samples are not so well labeled by the classifier, and the corresponding values are not so high. As a result, the median value of specificity or sensitivity taken across tissue samples is still high.

On the other hand, we get $I_{t}$ values of $r_{e}$. One value of $r_{e}$ results, as an average, from the prediction of 5 tissue samples. There is a significant probability that a sample difficult to predict is going to be represented in this set of 5 . Therefore, there is a significant probability that the average (i.e., $1-r_{e}$ ) will not be high.

The sixth experiment shows slightly better results than the fifth one on the $1-r_{e}$ score. The classification performance related to this experience is shown via a Receiver Operating Characteristic (ROC) curve (see Fig. 4).

\section{B. Extending the classification parameter set and the classi- fier set}

We now consider all Mueller parameters available (see section 3, Data preprocessing), and work with several standard classifiers. Among those classifiers, we still consider the decision stump on the scalar retardance at $450 \mathrm{~nm}$ used in the preceding section.

Fig. 5 shows an example of classification of an incoming tissue, using a decision stump on the linear retardance at wavelength $550 \mathrm{~nm}$ for the classifier. In the intensity image (element $m_{11}$ of the Mueller matrix, shown in Fig. 5-(a)), there is no noticeable difference between the healthy zones (two zones in the lower part of the image) and the three precancerous zones. Clear differences appear in the linear retardance image Fig. 5-(b). In this very simple case, the distributions $p(X \mid C)$ can be easily visualized on a simple graph, shown in Fig. 5-(c). By plotting histograms of pixels according to their ground truth $(C=3$ for precancerous and $C=4$ for healthy), the calculated retardance threshold appears as a frontier between the two groups. The difference between histograms in the training \& validation group (top) and the testing group (bottom) indicates the influence of inter-patients variability on the rather limited number of available samples.

In this study, a range of standard machine-learning strategies are applied. Their performances are compared using $1-r_{e}$ statistics given in Table 2 . In this table, for each family of classifiers two or more sets of values are presented, depending on the wavelength (for decision stump) or on the $\tau$-factor. $\tau$ represents the threshold used during variable selection according to the wrapper approach described earlier. Some classifiers are also given with a set of values for the discrete parameters at stake, among which the best value is retained. For example, classification trees used here could have 1 to 5 splits, the optimal number being determined by the algorithm itself (see section 4, Classification procedure).

For a better visualization of the results presented in Table 2 in terms of minimum, quartiles, maximum and average performances, a common boxplot representation is used on Fig. 6. From this figure, it becomes apparent that most of the classifiers have similar performances with a majority of runs having scores between $85 \%$ and $95 \%$. If we use the median value $q_{2}$ as a metric for choosing a classifier, the linear discriminant with a threshold 


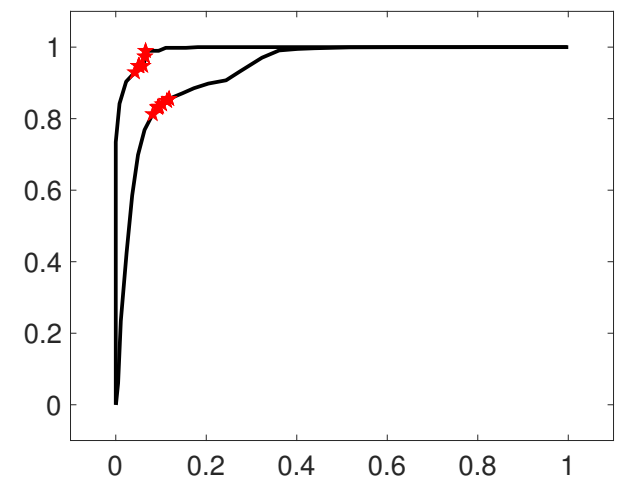

(a)

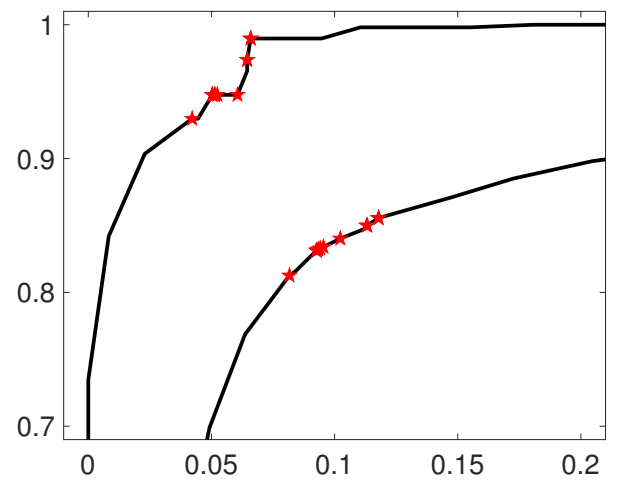

(b)

Fig. 4. ROC curve for experiment 6 . For any given threshold value, a decision is made on each superpixel of each of the 23 tissue samples; for each tissue, a True Positive Rate (TPR) is computed (if defined) and a False Positive Rate (FPR) is computed (if defined). For each threshold, with thus get a set of TPRs and a set of FPRs. In doing so, each tissue has the same weight, whereas averaging TPRs and FPRs across superpixels would give more weight to tissues having more superpixels. By computing the average value of each set, we get a point on the ROC curve (lower-right curve). We plot another curve (upper-left curve) by computing the median value of each set. The discrepancy between both curves (between average and median) shows that a few tissues are poorly predicted. The thresholds selected by the machine learning procedure proposed are plotted as dots on the curves (one dot for each test run). Subfigure (b): zoom on subfigure (a).

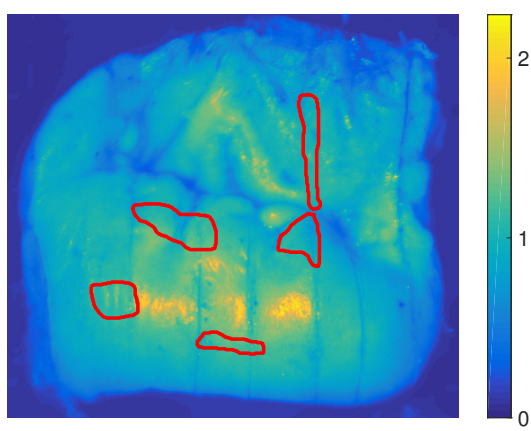

(a)
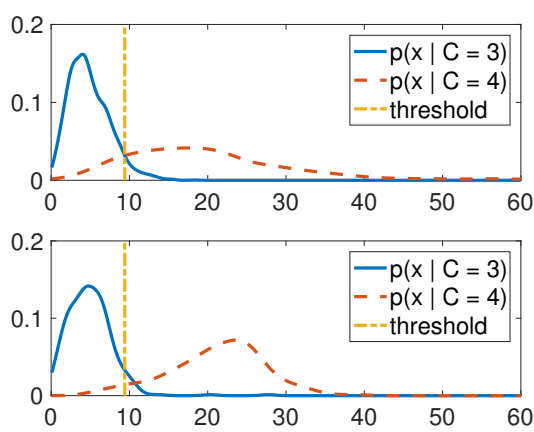

(c)

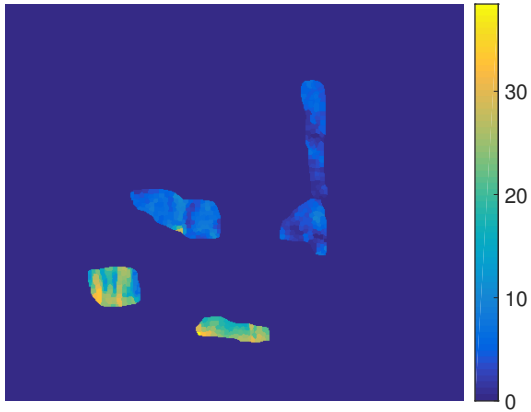

(b)

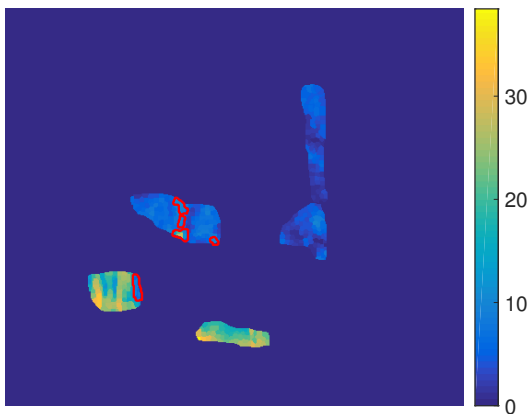

(d)

Fig. 5. Labeling of a given sample. Sample size: $459 \times 502$ pixels, $2.1 \times 2.3 \mathrm{~cm}$. Usual sample thickness is sample-dependent and may vary between 0.5 and $2 \mathrm{~cm}$, depending on the deepness of the lesion. Retained classifier: decision stump on the linear retardance, wavelength $550 \mathrm{~nm}$. Five zones were delineated by the clinician. The three uppermost zones in (a) correspond to precancerous tissue, the two lowest zones in (a) correspond to normal tissue. (a): intensity image with delineated zones; (b): linear retardance at $550 \mathrm{~nm}$ on the zones (linear retardance is here constant across superpixels); (c)-top: distributions $p$ (linear retardance $\mid C$ ) corresponding to the training \& validation data; (c)-bottom: distributions $p$ (linear retardance $\mid C$ ) for the testing data; (d): wrong decisions delineated in red (threshold: 9.5, as learnt for the training \& validation set of samples).

$\tau=1 \%$ (description (7)) yields what might be considered as the best results.
Experiment 7: we detail here the specificity and sensitivity scores of the linear discriminant with $\tau=1 \%$, to allow com- 
Table 2. Statistical Results for Some Standard Classifiers. Statistics Given Are Related to $100 \times\left(1-r_{e}\right)$. Unless Otherwise Mentioned, i.e., (1) and (2), All Parameters and All Wavelengths Were Considered.

\begin{tabular}{|c|c|c|}
\hline classifier & description & statistics: $\quad m\left[\begin{array}{lll}q_{1} & q_{2} & q_{3}\end{array}\right] M-a$; std IQR \\
\hline decision stump & (1) & 61.56 [ 86.5090 .1994 .13 ] 97.74 - $86.99 ; 9.987 .63$ \\
\hline decision stump & $(2)$ & 83.97 [ 88.8890 .7092 .98 ] 95.67 - $90.43 ; 3.004 .10$ \\
\hline decision stump & (3) & 63.37 [ 87.7590 .7092 .98 ] $95.67-89.29 ; 6.215 .23$ \\
\hline classification tree & $(4)$ & $60.72\left[\begin{array}{lll}85.90 & 88.9191 .36\end{array}\right] 95.66$ - $87.18 ; 8.145 .47$ \\
\hline classification tree & (5) & 63.37 [ 86.9689 .0992 .37 ] $95.73-88.44 ; 6.205 .41$ \\
\hline linear discriminant & (6) & $59.45\left[\begin{array}{lll}86.01 & 89.73 & 93.52\end{array}\right] 95.72$ - $87.90 ; 8.477 .51$ \\
\hline linear discriminant & (7) & $60.01\left[\begin{array}{lll}89.41 & 91.00 & 94.34\end{array}\right] 96.67$ - $89.86 ; 7.864 .93$ \\
\hline KNN & (8) & 64.52 [ 82.5787 .7690 .29 ] 94.36 - $85.39 ; 6.737 .72$ \\
\hline KNN & (9) & 71.30 [ 86.0590 .0492 .21 ] 95.69 - $87.92 ; 6.396 .16$ \\
\hline $\mathrm{KNN}$ & $(10)$ & 71.98 [ 87.9090 .3891 .71 ] 95.70 - $89.06 ; 4.983 .81$ \\
\hline naïve Bayes & $(11)$ & $54.26\left[\begin{array}{lll}85.04 & 89.95 & 92.34\end{array}\right] 94.11$ - $87.24 ; 8.847 .30$ \\
\hline naïve Bayes & $(12)$ & $55.68\left[\begin{array}{lll}87.23 & 90.27 & 93.05\end{array}\right] 95.98$ - $88.71 ; 8.225 .82$ \\
\hline random forest & (13) & 51.59 [ 86.6789 .1892 .73 ] 95.65 - $87.97 ; 8.526 .06$ \\
\hline random forest & $(14)$ & $63.37\left[\begin{array}{lll}86.44 & 89.82 & 92.40\end{array}\right] 95.93$ - $88.10 ; 7.295 .96$ \\
\hline SVM & (15) & $63.69\left[\begin{array}{llll}85.03 & 88.88 & 92.16\end{array}\right] 95.56$ - $87.17 ; 7.707 .13$ \\
\hline SVM & $(16)$ & $74.40\left[\begin{array}{lll}86.31 & 89.32 & 92.04\end{array}\right] 94.49$ - $88.51 ; 5.145 .72$ \\
\hline PLS & $(17)$ & $62.59\left[\begin{array}{lllll}75.24 & 84.07 & 88.48\end{array}\right] 94.80-81.03 ; 9.6113 .24$ \\
\hline PLS & $(18)$ & 52.30 [ 68.8378 .4683 .82 ] $90.92-76.67 ; 9.4414 .99$ \\
\hline
\end{tabular}

(1) scalar retardance @ 450 nm

(2) linear retardance @ $550 \mathrm{~nm}$ (see the remarkable values for $m$ and std)

(3) all parameters

(4) Maximum number of splits $\in\{1,2,3,4,5\} . \tau=0 \%$.

(5) Maximum number of splits $\in\{1,2,3,4,5\} \cdot \tau=1 \%$.

(6) Border $\in\{$ linear, quadratic $\} . \tau=0 \%$.

(7) Border $\in\{$ linear, quadratic $\}$. $\tau=1 \%$.

(8) Number of neighbors $\in\{1,3,5,7,9,11,13,15,17\} . \tau=0 \%$.

(9) Number of neighbors $\in\{1,3,5,7,9,11,13,15,17\} . \tau=1 \%$.

(10) Number of neighbors $\in\{1,3,5,7,9,11,13,15,17\} . \tau=6 \%$.

(11) $\tau=0 \%$.

(12) $\tau=1 \%$.

(13) 20 trees. Maximum number of splits $\in\{1,2,3,4\} . \tau=0 \%$.

(14) 20 trees. Maximum number of splits $\in\{1,2,3,4\} . \tau=1 \%$.

(15) $\tau=0 \%$. Gaussian kernel.

(16) $\tau=1 \%$. Gaussian kernel.

(17) A linear discriminant with a quadratic border is used in the latent space. Latent space dimension $\in\{1,2,3,5,8,10,15,20\} . \tau=0 \%$.

(18) A linear discriminant with a quadratic border is used in the latent space. Latent space dimension $\in\{1,2,3,5,8,10,15,20\} . \tau=1 \%$.

parison with experiments 5 and 6 . It may be noticed that the statistics on $1-r_{e}$ are improved.

\begin{tabular}{|l|c|c|c|c|c|c|}
\hline & $m$ & $q_{1}$ & $q_{2}$ & $q_{3}$ & $M$ & $a$ \\
\hline specificity & 51.2 & 80.4 & 93.7 & 99.3 & 100 & 87.9 \\
sensitivity & 50.0 & 92.2 & 95.7 & 99.6 & 100 & 90.7 \\
$1-r_{e}$ & 60.0 & 89.4 & 91.0 & 94.3 & 96.7 & 89.9 \\
\hline
\end{tabular}

It remains difficult, with the available database, to select an ideal classifier and the example here should be seen as an illustration of the use of the proposed framework. The main conclusion that may be drawn is that many classifiers yield results of similar quality, and that scores might be improved if using a perfectly labeled ground-truth. No general conclusion, such as optimal polarimetric parameter and best classification strategy, should be drawn from this study, even for the particular pathology of interest.

The proposed framework allows the comparison of a large number of machine-learning strategies and it should also be emphasized that the present work encompasses other comparable studies (Mueller imaging tissue diagnostic, [1, 11, 12]). It is well designed to work with a larger database, supports the addition of additional classes (low-grade cervical lesions, CIN 1-2, metaplasia etc.) and the seamless integration of meta-data concerning the patients (age, smoking etc.). Transposition to the application of polarimetry to other pathologies is straightforward. 


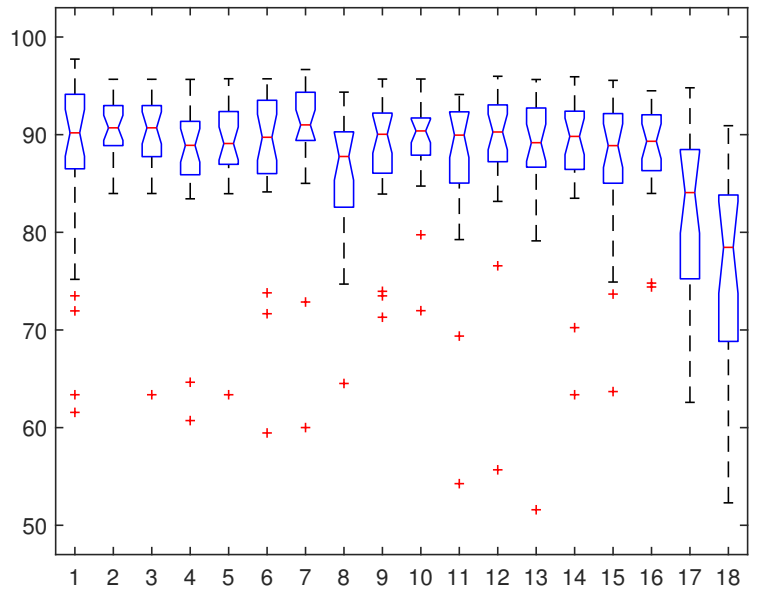

Fig. 6. Boxplots of the statistics stemming from the classification procedures considered (see Table 2). X-labels correspond to indexes of the procedures (description column in Table 2).

\section{CONCLUSION}

In this paper, a principled statistical analysis approach has been proposed for a Mueller polarimetric clinical study. The proposed framework comprises (1) a complete preprocessing pipeline, including physically admissible retrieval of Mueller parameters, calculation of polarimetric parameters through Lu-Chipman decomposition, and dimension reduction through superpixels; (2) a decision-theoretic classification procedure, allowing to automatically select a classifier among a given set, to determine its parameters, and to extract the relevant polarimetric parameters to be considered.

The framework was applied on a previously partially published database of 23 conization pieces. We showed that using superpixels in the preprocessing step improves results. Among a set of widely used classification algorithms (decision stumps, classification trees, linear discriminant, KNN, naïve Bayes, random forest, SVM, and PLS), a number of approaches yielded similar results, with scores ranging from $85 \%$ to $95 \%$. A linear discriminant classifier was the best suited to the available database, outperforming strategies proposed in earlier works (and encompassed in this study). However, no definite conclusion as to for example the best classifier to be used can be drawn from the present study. This task would require the definition of the best tradeoff between performance and algorithm complexity, and would necessitate a larger tissue database.

With an increasing number of studies resorting to Mueller polarimetric imaging, in particular in biological tissue diagnosis, there is a need of principled statistical analysis approaches. Those were lacking in this field. We hope this article will contribute bridging this gap.

Finally, the procedure will be used on other pathologies, with more than two labels, and using a cost different from the Kronecker delta used here. This is left for future work.

\section{APPENDIX : CLASSIFICATION PROCEDURE: A LINK WITH CONFUSION MATRICES}

A confusion matrix point of view on the proposed classification procedure is given in this section. No additional methodological points are brought here. The goal is to link the approach with specificity and sensitivity scores, as well as with classification accuracy and average class accuracy, which are classically used in classifier assessment.
The goal is to differentiate normal tissue (in this study, healthy squamous epithelium (EMS)) labeled $c=0$, from abnormal tissue (in this study, high-grade cervical intraepithelial neoplasia (CIN 2-3)) labeled $c=1$. Let us consider three tissue samples, numbered say $i_{s} \in\{5,6,7\}$, on which predictions have been made. This set of indexes has been chosen to differ from the class indexes 0 and 1, so as to avoid any confusion. These predictions are supposed to take place in the $i_{t}^{\text {th }}$ test run. The corresponding confusion matrices, for tissue types 0 (first raw and first column in the matrices) and 1 (second raw and second column in the matrices), are supposed to write

$$
\left[\begin{array}{ll}
\alpha_{5} & \beta_{5} \\
\gamma_{5} & \delta_{5}
\end{array}\right] \quad\left[\begin{array}{cc}
\alpha_{6} & \beta_{6} \\
0 & 0
\end{array}\right] \quad\left[\begin{array}{cc}
0 & 0 \\
\gamma_{7} & \delta_{7}
\end{array}\right] .
$$

Let $n_{0}=\alpha_{5}+\beta_{5}+\alpha_{6}+\beta_{6}$ and $n_{1}=\gamma_{5}+\delta_{5}+\gamma_{7}+\delta_{7}$ be respectively the total number of superpixels labeled 0 and 1 in the ground-truth. Let $n=n_{0}+n_{1}$ be the total number of superpixels involved.

The corresponding conditional empirical risks write

$$
\begin{aligned}
& r_{e}(5 \mid 0)=\left(\alpha_{5} \ell(0,0)+\beta_{5} \ell(0,1)\right) /\left(\alpha_{5}+\beta_{5}\right) \\
& r_{e}(5 \mid 1)=\left(\gamma_{5} \ell(1,0)+\delta_{5} \ell(1,1)\right) /\left(\gamma_{5}+\delta_{5}\right) \\
& r_{e}(6 \mid 0)=\left(\alpha_{6} \ell(0,0)+\beta_{6} \ell(0,1)\right) /\left(\alpha_{6}+\beta_{6}\right) \\
& r_{e}(7 \mid 1)=\left(\gamma_{7} \ell(1,0)+\delta_{7} \ell(1,1)\right) /\left(\gamma_{7}+\delta_{7}\right) \\
& r_{e}(6 \mid 1) \quad \text { undefined } \\
& r_{e}(7 \mid 0) \quad \text { undefined }
\end{aligned}
$$

Classical assessment scores are

$$
\begin{array}{ll}
\text { classification accuracy: } & \left(\alpha_{5}+\alpha_{6}+\delta_{5}+\delta_{7}\right) / n \\
\text { average class accuracy: } & \frac{1}{2}\left(\left(\alpha_{5}+\alpha_{6}\right) / n_{0}\right. \\
& \left.+\left(\delta_{5}+\delta_{7}\right) / n_{1}\right) \\
& \left(\alpha_{5}+\alpha_{6}\right) / n_{0} \\
\text { specificity: } & \left(\delta_{5}+\delta_{7}\right) / n_{1}
\end{array}
$$

The major problem regarding the use of classification accuracy as an assessment score appears in the case of imbalanced classes. If label $c=1$ is underrepresented in the ground-truth, classification accuracy is overwhelmingly determined by the labeling of superpixels with ground-truth $c=0$. A classifier labeling each superpixel with 0 would then yield a high assessment score, but would not constitute a pertinent choice, since all abnormal superpixels would be missed. 
Imbalanced tissue samples can also be an obstacle towards devising an appropriate classifier. In the example taken, tissue sample 7 could for example constitute a point of view on the random variables $(X, C=1)$ different and complementary to the point of view conveyed by the superpixels in tissue sample 5 , which are samples of $(X, C=0)$ and of $(X, C=1)$. If the number of superpixels in tissue sample 7 is negligible as compared to the number of superpixels in tissue sample 5 (i.e., more precisely if $\delta_{5}+\gamma_{5} \gg \delta_{7}+\gamma_{7}$ ), the information conveyed by sample 7 will be left unseen by the procedure. The assessment score will focus on the point of view of tissue sample 5, and if a fresh tissue sample similar to tissue sample 7 appears, the classifier selected by the assessment score will yield a labeling akin to the features of the superpixel population in 5, which is troublesome as the fresh tissue should be evaluated in the light of the superpixels of 7 .

The problem related to classification accuracy vanishes with the use of average class accuracy, where all classes have the same weight, regardless of the number of superpixels at stake in each class.

Tissue sample imbalance can be remedied using the following assessment score

$$
\frac{1}{2}\left[\frac{1}{2}\left(\frac{\alpha_{5}}{\alpha_{5}+\beta_{5}}+\frac{\alpha_{6}}{\alpha_{6}+\beta_{6}}\right)+\frac{1}{2}\left(\frac{\delta_{5}}{\delta_{5}+\gamma_{5}}+\frac{\delta_{7}}{\delta_{7}+\gamma_{7}}\right)\right]
$$

which also handles imbalanced classes.

The preceding scores may be interpreted in a probabilistic framework. The probability of correct classification writes

$$
\begin{aligned}
& p(\widehat{C}=0, C=0)+p(\widehat{C}=1, C=1) \\
& =p(\widehat{C}=0 \mid C=0) p(C=0) \\
& +p(\widehat{C}=1 \mid C=1) p(C=1) .
\end{aligned}
$$

The corresponding empirical quantities, denoted using $\mathcal{E}($.$) ,$ write

$$
\begin{aligned}
\mathcal{E}(p(\widehat{C}=0 \mid C=0)) & =\left(\alpha_{5}+\alpha_{6}\right) / n_{0}=\text { specificity } \\
\mathcal{E}(p(C=0)) & =n_{0} / n \\
\mathcal{E}(p(\widehat{C}=1 \mid C=1)) & =\left(\delta_{5}+\delta_{7}\right) / n_{1}=\text { sensitivity } \\
\mathcal{E}(p(C=1)) & =n_{1} / n
\end{aligned}
$$

As a consequence, classification accuracy may be interpreted as the empirical probability of correct classification, and average class accuracy happens to be interpreted as the empirical probability of correct classification, with the prior class probability distribution forced to the uniform distribution. This writes

average class accuracy $=$

$$
\begin{aligned}
& \mathcal{E}(p(\widehat{C}=0 \mid C=0)) p(C=0) \\
& +\mathcal{E}(p(\widehat{C}=1 \mid C=1)) p(C=1),
\end{aligned}
$$

where $p(C=0)=p(C=1)=\frac{1}{2}$ is enforced. It happens that Eq. (12) may be interpreted as an expression akin to average class accuracy (see the right hand side of Eq. (15)), with an evaluation of $\mathcal{E}(p(\widehat{C}=0 \mid C=0))$ differing from Eqs. (14a) - (14d), the evaluation of $\mathcal{E}(p(\widehat{C}=1 \mid C=1))$ having been adapted as well.
Those quantities will now be linked to the empirical risks at stake in the proposed approach. The empirical risk for the $i_{t}^{\text {th }}$ test run writes

$$
\begin{aligned}
r_{e}= & {\left[\left(r_{e}(5 \mid 0)+r_{e}(6 \mid 0)\right) / 2\right] p(C=0) } \\
& +\left[\left(r_{e}(5 \mid 1)+r_{e}(7 \mid 1)\right) / 2\right] p(C=1) .
\end{aligned}
$$

In the present study, we chose $\ell(a, b)=1-\delta_{a, b}$ and $p(C=0)=$ $p(C=1)=\frac{1}{2}$. Other choices are possible and even advocated for $\ell$, as mentioned in the article. But those particular choices allow simple and direct comparison to classical procedures, as will now be shown. This was the very reason motivating these choices.

With

$$
\begin{aligned}
\left\langle r_{e}\left(i_{s} \mid 0\right)\right\rangle_{i_{s} \in\{5,6,7\}} & =\frac{1}{2}\left(r_{e}(5 \mid 0)+r_{e}(6 \mid 0)\right) \\
& =\frac{1}{2}\left(\frac{\beta_{5}}{\alpha_{5}+\beta_{5}}+\frac{\beta_{6}}{\alpha_{6}+\beta_{6}}\right) \\
\left\langle r_{e}\left(i_{s} \mid 1\right)\right\rangle_{i_{s} \in\{5,6,7\}} & =\frac{1}{2}\left(r_{e}(5 \mid 1)+r_{e}(7 \mid 1)\right) \\
& =\frac{1}{2}\left(\frac{\gamma_{5}}{\gamma_{5}+\delta_{5}}+\frac{\gamma_{7}}{\gamma_{7}+\delta_{7}}\right),
\end{aligned}
$$

the empirical risk $r_{e}$ boils down to

$$
r_{e}=\left(\frac{\beta_{5}}{\alpha_{5}+\beta_{5}}+\frac{\beta_{6}}{\alpha_{6}+\beta_{6}}\right) \frac{1}{4}+\left(\frac{\delta_{5}}{\delta_{5}+\gamma_{5}}+\frac{\delta_{7}}{\delta_{7}+\gamma_{7}}\right) \frac{1}{4}
$$

Minimizing $r_{e}$ amounts to maximizing $1-r_{e}$, which writes

$$
\begin{aligned}
& \frac{1}{4} \frac{\alpha_{5}+\beta_{5}}{\alpha_{5}+\beta_{5}}+\frac{1}{4} \frac{\alpha_{6}+\beta_{6}}{\alpha_{6}+\beta_{6}}+\frac{1}{4} \frac{\delta_{5}+\gamma_{5}}{\delta_{5}+\gamma_{5}}+\frac{1}{4} \frac{\delta_{7}+\gamma_{7}}{\delta_{7}+\gamma_{7}}-r_{e} \\
& =\frac{1}{4} \frac{\alpha_{5}}{\alpha_{5}+\beta_{5}}+\frac{1}{4} \frac{\alpha_{6}}{\alpha_{6}+\beta_{6}}+\frac{1}{4} \frac{\delta_{5}}{\delta_{5}+\gamma_{5}}+\frac{1}{4} \frac{\delta_{7}}{\delta_{7}+\gamma_{7}},
\end{aligned}
$$

and it happens that our approach ends up maximizing expression (12), with all its nice properties (i.e., imbalanced classes and imbalanced tissues are take care of). Those properties will held with other choices for $\ell$.

Finally, let us mention that for a given classifier the risk $\mathcal{R}$ also writes

$$
\mathcal{R}=\sum_{a, b} \ell(a, b) p(C=a) p(\widehat{C}=b \mid C=a) .
$$

This expression highlights the link between the prior $p(C=a)$ and the cost $\ell(a, b)$, which appear through their product. Loss and prior should therefore be analyzed simultaneously [24].

This work was funded by the Institut National du Cancer (INCa) and the Cancéropôle (2012-1-GYN-01-EP-1). The study was conducted under the supervision of the INCa and Cancéropôle who guaranteed that the internationally accepted principles and practices related to the ethical conduct of research involving the use of human subjects or animals were observed in this study.

\section{ACKNOWLEDGMENTS}

The authors are grateful to the anonymous reviewers for their useful comments. 


\section{REFERENCES}

1. J. Rehbinder, H. Haddad, S. Deby, B. Teig, A. Nazac, T. Novikova, A. Pierangelo, and F. Moreau, "Ex vivo Mueller polarimetric imaging of the uterine cervix: a first statistical evaluation," J. Biomed. Opt. 21 (2016).

2. J. Vizet, J. Rehbinder, S. Deby, S. Roussel, A. Nazac, R. Soufan, C. Genestie, C. Haie-Meder, H. Fernandez, F. Moreau, and A. Pierangelo, "In vivo imaging of uterine cervix with a Mueller polarimetric colposcope," Sci. Reports 7, 2471 (2017).

3. V. Tuchin, "Polarized light interaction with tissues," J. Biomed. Opt. 21, 071114 (2016).

4. N. Ghosh and A. Vitkin, "Tissue polarimetry: concepts, challenges, applications, and outlook," J. Biomed. Opt. 16, 110801 (2011).

5. M. Gurcan, L. Boucheron, A. Can, A. Madabhushi, N. Rajpoot, and B. Yener, "Histopathological image analysis: a review," IEEE Rev. Biomed. Eng. 2, 147-171 (2009).

6. A. Alenin, L. Morrison, C. Curiel, and J. S. Tyo, "Hyperspectral measurement of the scattering of polarized light by skin," in Polarization Science and Remote Sensing V, vol. 8160 of Proceedings of SPIE (2011), p. 816014

7. B. Hoover and J. S. Tyo, "Polarization components analysis for invariant discrimination," Appl. Opt. 46, 8364-8373 (2007).

8. G. Anna, F. Goudail, and D. Dolfi, "Optimal discrimination of multiple regions with an active polarimetric imager," Opt. Express 19, 2536725378 (2011).

9. G. Anna, F. Goudail, and D. Dolfi, "Polarimetric target detection in the presence of spatially fluctuating Mueller matrices," Opt. Lett. 36, 4590-4592 (2011).

10. I. Vaughn, B. Hoover, and J. S. Tyo, "Classification using active polarimetry," in Polarization: Measurement, Analysis, and Remote Sensing X, , vol. 8364 of Proceedings of SPIE (2012), p. 83640S.

11. I. Ahmad, M. Ahmad, K. Khan, S. Ashraf, S. Ahmad, and M. Ikram, "Ex vivo characterization of normal and adenocarcinoma colon samples by Mueller matrix polarimetry," J. Biomed. Opt. 20, 056012 (2015).

12. J. Wang, W. Zheng, K. Lin, and Z. Huang, "Integrated Mueller-matrix near-infrared imaging and point-wise spectroscopy improves colonic cancer detection," Biomed. Opt. Express 7, 1116-1126 (2016).

13. V. Ushenko, M. Sidor, Y. Marchuk, N. Pashkovskaya, and D. Andreichuk, "Mueller-matrix mapping of biological tissues in differential diagnosis of optical anisotropy mechanisms of protein networks," Quantum Electron. 45, 265-269 (2015).

14. Y. Xu, J.-Y. Zhu, E. I-Chao, M. Lai, and Z. Tu, "Weakly supervised histopathology cancer image segmentation and classification," Med. Image Analysis 18, 591-604 (2014).

15. A. Pierangelo, A. Nazac, A. Benali, P. Validire, H. Cohen, T. Novikova, B. Ibrahim, S. Manhas, C. Fallet, M.-R. Antonelli, and A. De Martino, "Polarimetric imaging of the uterine cervix: a case study," Opt. Express 21 (2013).

16. S. Faisan, C. Heinrich, G. Sfikas, and J. Zallat, "Estimation of Mueller matrices using non-local means filtering," Opt. Express 21, 4424-4438 (2013).

17. R. Achanta, A. Shaji, K. Smith, A. Lucchi, P. Fua, and S. Süsstrunk, "SLIC superpixels," Tech. Rep. 149300, EPFL (2010).

18. R. Achanta, A. Shaji, K. Smith, A. Lucchi, P. Fua, and S. Süsstrunk, "SLIC superpixels compared to state-of-the art superpixel methods," IEEE Transactions on Pattern Analysis Mach. Intell. 34, 2274-2281 (2012).

19. J. Berger, Statistical decision theory and Bayesian analysis (Springer, 1985, chap. 1, sec. 5, pp. 16-17; chap. 2, sec. 4, pp. 64-68), 2nd ed.

20. C. Bishop, Pattern recognition and machine learning (Springer, 2006, chap. 1, sec. 5, pp. 38-48).

21. T. Hastie, R. Tibshirani, and J. Friedman, The elements of statistical learning: data mining, inference, and prediction (Springer, 2009, ch. 7, sec. 2, p. 222), 2nd ed.

22. Y. Liu and W. Rayens, "PLS and dimension reduction for classification," Comput. Stat. 22, 189-208 (2007).

23. M. Barker and W. Rayens, "Partial least squares for discrimination," J. Chemom. 17, 166-173 (2003).
24. C. Robert, The Bayesian choice: from decision-theoretic foundations to computational implementation (Springer, 2007, ch. 2, sec. 1, pp. 5253), 2nd ed. 